\title{
A GIANT ARGON BUBBLE CHAMBER WITH LONG SENSITIVE TIME FOR NUCLEON DECAY EXPERIMENTS
}

\author{
Gert G. HARIGEL \\ CERN, Geneva, Switzerland
}

\begin{abstract}
A giant argon bubble chamber could be a suitable detector to investigate the decay modes of nucleons in an unbiased way. We are investigating the possibility of operating such a chamber with a track sensitive duty cycle of up to $\sim 50 \%$. A small and not very clean bubble chamber has been successfully operated in an expanded pressure stabilized mode with a duty cycle of $10 \%$. A tentative design of a $3000 \mathrm{~m}^{3}$ argon bubble chamber is discussed.
\end{abstract}

A giant bubble chamber, containing several thousand tons of liquid argon, could be a suitable detector for nucleon decays, because argon is available in unlimited quantity, it is non-inflammable, and it can be cooled by liquid nitrogen, making the detector filling cheap and relatively safe in operation.

The main advantages of argon are that it can be used as track sensitive bubble chamber liquid and that the scintillation light, produced by charged particles, can be employed as a trigger for the illumination system of the chamber. The well-known advantages of bubble chambers are also present in argon; momentum measurement from range, particle identification and determination of the direction of flight from ionization via bubble density.

Evidently, for such a new generation experiment, the following requirements should be fulfilled first: (1) the proton decay has to be firmly established, (2) its lifetime should not be larger than $10^{32}$ years, (3) the detector must have a large duty cycle and (4) should contain the decay products, (5) the scintillation light should enable triggering on decays and discriminate against the cosmic muon background. Then, together with the determination of the charge from curvature in the magnetic field, the decay channels of the proton can be investigated.

Prior to any detailed design of such a detector (preliminary ideas are published [1]), tests have to be made in a fairly large chamber, such as BEBC, since results from our 2.7 liter test apparatus do not yet answer completely all technical questions. This concerns in particular the duty cycle, probably limited by parasitic boiling rather than by throughgoing particles, as well as the construction of the expansion bellows. A choice between bright-field and holographic illumination has to be made. The optical transparency of the liquid, specifically in view of possible absorption of the scintillation light, has to be studied. The selection of a deep underground site is governed by various aspects; seismics and stability of the rock, size of the access shaft, assembly of components, safety vents and transfer lines, etc. However, at present we do not see any insurmountable problems for the construction of such a detector.

\section{Reference}

[1] G. Harigel, A. Hervé and K. Winter, Nucl. Instr. and Meth. $216(1983) 355$ 